\title{
DPPX Modifies TEA Sensitivity of the Kv4 Channels in Rabbit Carotid Body Chemoreceptor Cells.
}

O. COLINAS, F.D. PÉREZ-CARRETERO, E. ALONSO, J.R. LÓPEZLÓPEZ and M. T. PÉREZ-GARCÍA.

Departamento de Bioquímica y Biología Molecular y Fisiología e Instituto de Biología y Genética Molecular (IBGM), Universidad de Valladolid y Consejo Superior de

Investigaciones Científicas (CSIC), c/Sanz y Forés s/n, 47003 Valladolid, Spain.

\begin{abstract}
Chemoreceptor cells from rabbit carotid body (CB) exhibit transient outward currents reversibly inhibited by low $\mathrm{P}_{\mathrm{O} 2}$. Molecular and functional dissection of the components of these outward currents indicates that at least two different channels (Kv4.3 and Kv3.4) contribute to this current. Furthermore, several lines of evidence support the conclusion that Kv4 channel subfamily members (either $\mathrm{Kv} 4.3$ alone or $\mathrm{Kv} 4.3 / \mathrm{Kv} 4.1$ heteromultimers) are the oxygen sensitive $\mathrm{K}$ channels $\left(\mathrm{K}_{\mathrm{O} 2}\right)$ in rabbit $\mathrm{CB}$ chemoreceptor cells. However, the pharmacological characterization of these currents shows that they are almost completely blocked by high external TEA concentrations, while Kv4 channels have been shown to be TEA-insensitive. We hypothesized that the expression of regulatory subunits in chemoreceptor cells could modify TEA sensitivity of Kv4 channels. Here, we explore the presence and functional contribution of DPPX to $\mathrm{K}_{\mathrm{O} 2}$ currents in rabbit $\mathrm{CB}$ chemoreceptor cells by using DPPX functional knockdown with siRNA. Our data suggest that DPPX proteins are integral components of $\mathrm{K}_{\mathrm{O} 2}$ currents, and that their association with Kv4 subunits modulate the pharmacological profile of the heteromultimers.
\end{abstract}

\section{INTRODUCTION}

In rabbit carotid body chemoreceptor cells, genes of the Kv4 family (either Kv4.3 alone or Kv4.3/Kv4.1 heteromultimers) have been shown to represent the molecular correlate of the oxygen-sensitive voltage dependent $\mathrm{K}^{+}$current $\left(\mathrm{K}_{\mathrm{O} 2}\right)$ originally described in this preparation (Perez-Garcia et al., 2000; Sanchez et al., 2002;López-López et al., 2003).

The variability observed in gating kinetics and conductance among the Kv4 mediated native current does not seem to affect the pharmacological profile of Kv4 currents, which are typically described as 4-AP sensitive and TEAresistant. This holds true when characterizing Kv4 currents in heterologous expression systems (Pak et al., 1991;Jerng \& Covarrubias, 1997) and also when studying native currents (Martina et al., 1998;Song et al., 1998), suggesting that the association of Kv4 pore-forming subunits with accessory subunits does not change the pharmacological properties of the heteromultimers. However, in rabbit $\mathrm{CB}$ chemoreceptor cells we have 
observed that transient outward $\mathrm{K}^{+}$currents are sensitive to 4-AP (LópezLópez et al., 1993) and heteropodatoxin (HpTx-2), (Sanchez et al., 2002), but can also be blocked by external TEA application. While we have identified $\mathrm{Kv} 3.4$ as the high sensitive TEA component of the transient $\mathrm{K}^{+}$current (Kaab et al., 2005; Sanchez et al., 2002), we have no explanation for the blockade of the Kv4 component with low-millimolar TEA concentrations. In the search for an explanation of this perplexing observation, we have explored the possibility that some accessory subunit of Kv4 channels could determine the atypical pharmacological profile of the transient $\mathrm{K}^{+}$current in rabbit $\mathrm{CB}$ chemoreceptor cells. Among Kv4 auxiliary subunits, the structural properties of DPPX, with a large C-terminal extracellular domain (Wada et al., 1992), made conceivable the hypothesis that its association with $\mathrm{Kv} 4 \alpha$ subunits could modify the binding of TEA to the external side of the pore. We detected high expression levels of DPPX mRNA in rabbit CB chemoreceptor cells (when compared to cerebellar granule neurons), DPPX protein was found to co-express with Kv4.3 in this preparation, and its functional knockdown with siRNA decreased its sensitivity to extracellular TEA, demonstrating the physiological association in a native tissue.

\section{METHODS}

\subsection{Dissociation and Culture of Rabbit Carotid Body Cells}

Adult New Zealand rabbits $(1.5-2 \mathrm{~kg})$ were anesthetized with intravenous application of sodium pentobarbital $(40 \mathrm{mg} / \mathrm{kg})$ through the lateral vein of the ear. After tracheostomy, carotid artery bifurcations were dissected out and animals were killed by intracardiac injection of sodium pentobarbital. The CBs were enzymatically dispersed as previously described (Pérez-García et al., 1992). Dispersed cells were plated onto poly-L-lysine-coated coverslips with $2 \mathrm{ml}$ of growth medium, and maintained in culture at $37^{\circ} \mathrm{C}$ in a $5 \% \mathrm{CO}_{2}$ atmosphere up to $96 \mathrm{~h}$.

\subsection{Electrophysiological Methods}

Ionic currents were recorded at room temperature $\left(20-25^{\circ} \mathrm{C}\right)$ using the wholecell configuration of the patch-clamp technique. Whole-cell current recordings and data acquisition from $\mathrm{CB}$ chemoreceptor cells were made as previously described (López-López et al., 1997;Sanchez et al., 2002).

\section{3. siRNA Design and Construction}

We selected a target sequence for designing siRNA against rabbit DPPX in a region with the highest identity with the human sequence. The siRNA sequence was 5'-ACACGAGGATGAAAGTGAA-3'. After using BLAST program to ensure specificity of the sequences across mammalian genomes, the target sequence was used to generate siRNA Expression Cassette (SEC) with the Silencer ${ }^{\mathrm{TM}}$ Express (siRNA Expression Cassette Kit, Ambion) 
DPPX Modifies TEA Sensitivity of the Kv4 Channels in Rabbit Carotid Body Chemoreceptor Cells.

according to the manufacturer's instructions. A negative control SEC with limited homology to the mammalian genomes was used as control.

\subsection{RNA Isolation and RT-PCR Methods}

The expression levels of DPPX mRNA in CB chemoreceptor cells were determined from total RNA extracted from pooled chemoreceptor cells kept in culture during 24-48h. Control experiments were performed in cultured cerebellar granule cells obtained from rabbit cerebellum according to previously published protocols (Liu et al., 2007) and kept in culture for 5-7 days. Electrodes made from capillary glass baked overnight at $200{ }^{\circ} \mathrm{C}$ were filled with $7 \mu \mathrm{l}$ of RNAse-free water and the tip was broken to facilitate the aspiration of multiple cells. After collecting 25-50 cells (CB chemoreceptor cells or cerebellar granule cells), the contents of the pipette were ejected into a $0.2 \mathrm{ml}$ eppendorf tube containing $1 \mu \mathrm{l}$ of RNAsin $(20 \mathrm{u} / \mu \mathrm{l}$, Applied Biosystems) and kept at $-80^{\circ} \mathrm{C}$. RNA was reverse transcribed with MuLvRT $(5000 \mathrm{u} / \mathrm{ml})$ as previously described (Kaab et al., 2005).

Total mRNA from rabbit hippocampus or cerebellum using TRIzol Reagent was used as the calibrator.

The mRNA levels for DPPX were determined by quantitative real-time PCR (qPCR) on a Rotor-Gene 3000 instrument (Corbett Research) using ribosomal protein L18 (RPL18) expression levels as housekeeping gene. Amplification of cDNA was performed as previously described (Kaab et al., 2005). The PCR primers were designed using the Primer 3 website (http://frodo.wi.mit.edu/cgi-bin/primer3/primer3_www.cgi) and were selected to spam an intronic sequence and to recognize both human and rabbit DPPX. The primer sequences were 5', CACGAGGATGAAAGTGAACG 3 ' (forward) and 5', TGATGGACTGGATGTTGTCG 3' (reverse), and they amplify a $178 \mathrm{bp}$ fragment.

The data were analyzed using the threshold cycle $(\mathrm{Ct})$ relative quantification method, (Livak \& Schmittgen, 2001) so that the fold change in expression ( $2^{-\Delta \Delta \mathrm{Ct}}$ ) was calculated from $\Delta \Delta \mathrm{Ct}$ values obtained with the expression

$$
\Delta \Delta \mathrm{Ct}=\left(\mathrm{Ct}_{\mathrm{DPPX}}-\mathrm{Ct}_{\mathrm{RPL18}}\right)_{\mathrm{CBcells}}-\left(\mathrm{Ct}_{\mathrm{DPPX}}-\mathrm{Ct}_{\mathrm{RPL18}}\right)_{\text {Hippocampus }}
$$

Using the $2^{-\Delta \Delta \mathrm{Ct}}$ method, the data in CB cells and $\mathrm{G}$ cells are presented as the fold change in gene expression normalized to RPL18 and relative to hippocampus.

\subsection{Electroporation of Cultured Rabbit CB Chemoreceptor Cells}

Single cell electroporation was performed using modified patch-clamp techniques, following previously published models (Rae \& Levis, 2002). A home-made device consisting on an operational amplifier connected to a current to voltage converter allows to supply train pulses from -9 to $+9 \mathrm{~V}$ through a patch pipette. The train pulses were produced using pClamp an Digidata 1200 as the pulse generator, which allows independent setting of the 
duration and frequency of each pulse and the total time of the train pulse delivery. The protocol used and the electroporation pipettes were made as previously described (Colinas et al., 2008). The pipette solution was the internal solution used for the recording experiments to which $33 \mathrm{ng} / \mu \mathrm{l}$ of a plasmid DNA encoding GFP alone or in combination with $7 \mathrm{ng} / \mu \mathrm{l}$ of DPPX or negative control SEC were added. GFP-transfected cells were recorded 24 to $48 \mathrm{~h}$ after electroporation.

\subsection{Immunocytochemical Methods}

Immunocytochemistry on isolated CB chemoreceptor cells in culture was performed as described previously (Perez-Garcia et al., 2004).

\section{RESULTS}

\subsection{TEA Effect on Transient $\mathrm{K}^{+}$Currents From Rabbit CB Chemoreceptor Cells}

We tested the effect of TEA on the native CB chemoreceptor cells by analyzing the reduction in the peak current amplitude in $200 \mathrm{~ms}$ depolarizing pulses to $+40 \mathrm{mV}$ in the presence of increasing TEA concentrations (from 0.1 $\mu \mathrm{M}$ to $100 \mathrm{mM}$ ) in the bath solution. The best fit to the data from $12 \mathrm{CB}$ chemoreceptor cells was obtained with the three binding-site model with affinity constants obtained of $3.4 \mu \mathrm{M}, 130 \mu \mathrm{M}$ and $24 \mathrm{mM}$ and relative amplitudes of $8.1,62.64$ and $12.26 \%$ respectively. In addition to this three components, there is a non-inactivating component that was unaffected by high concentrations of TEA representing a $17 \%$ of the total current. In all the cases, the best fit to the data from each individual cell was also obtained with the three binding-site model. The dose-response curve obtained in one cell is depicted in Figure 1A. The relative amplitude of these three components in the example shown was $14 \%, 45 \%$ and $16 \%$ respectively. The TEA resistant fraction averaged $24 \%$. The dissociation constants $(\mathrm{Kd})$ are also indicated on the figure.

Transient outward currents in rabbit CB chemoreceptor cells have been shown to be comprised of Kv3.4 and Kv4.1/4.3 channels (Sanchez et al., 2002;Perez-Garcia et al., 2000), and both the pharmacological and kinetic profile are consistent identifying Kv3.4 as the high-affinity TEA component. However, the intermediate affinity (and to a lower extent the low-affinity) component is clearly a transient current representing the larger portion of the total outward current, that can be attributable to Kv4 currents, and that shows an atypical behavior with respect to TEA sensitivity. In order to explore more directly this extent, we investigated if selective blockers of Kv4 channels, such as heteropodatoxin-2 (Sanguinetti et al., 1997), inhibit the same channel population than submillimolar TEA concentrations. We have used $50 \mu \mathrm{M}$ TEA to block completely the high TEA-sensitivity component, that we have previously described as the BDS-sensitive component (Kaab et al., 2005), and TEA $500 \mu \mathrm{M}$ to block the intermediate TEA sensitivity component. In this set of experiments (see Figure 1B), CB chemorreceptor cells were 
DPPX Modifies TEA Sensitivity of the Kv4 Channels in Rabbit Carotid Body Chemoreceptor Cells.

subjected to two consecutive applications of $1 \mu \mathrm{M} \mathrm{HpTx}$, in the presence of 50 and $500 \mu \mathrm{M}$ TEA respectively.

A
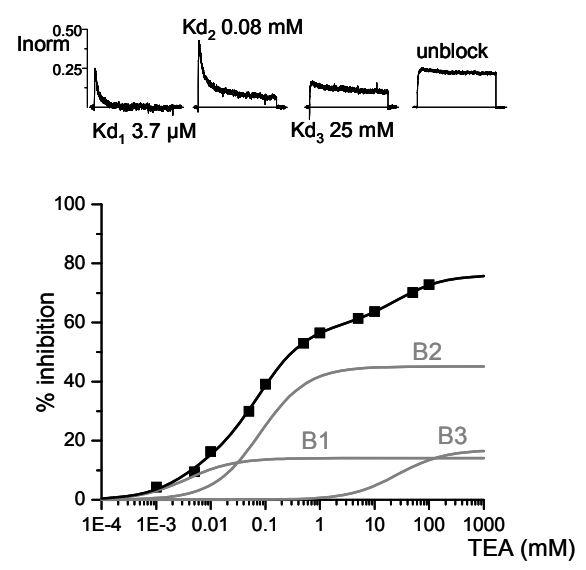

B
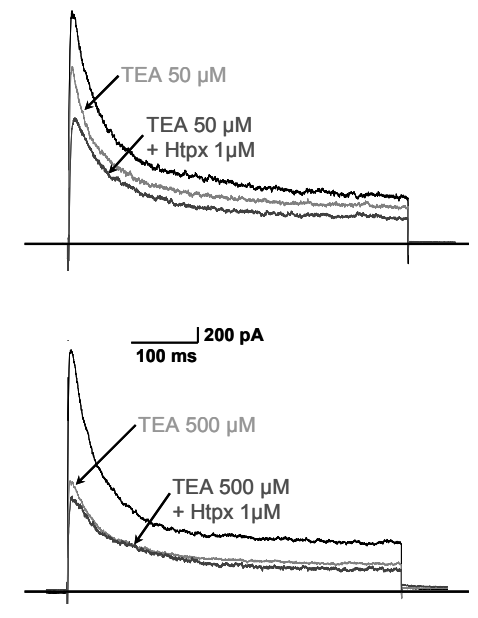

Figure 1. TEA effect on transient $\mathrm{K}^{+}$currents from rabbit chemoreceptor cells. A) TEA dose-response curve obtained with data from one $\mathrm{CB}$ chemoreceptor cell. The continuous line through the data points shows the best fit obtained with a hyperbolic function with three binding sites. The functions representing the three components are also shown in the figure (B1,B2 and B3). The upper panels in the figure show normalized traces obtained by subtracting the traces in the presence of $5 \mu \mathrm{M}, 100 \mu \mathrm{M}$ and $25 \mathrm{mM}$ TEA from the control traces, to get an estimation of the overall shape of the currents blocked by TEA concentrations closed to the obtained affinity constants ( $\mathrm{Kd}$ indicated in each trace). The unblock trace is the current remaining in the presence of 100 mM TEA. B) Traces show representative examples of depolarizing pulses to $+40 \mathrm{mV}$ in control or induced by the application of $50 \mu \mathrm{M}$ TEA (upper panel) or $500 \mu \mathrm{M}$ TEA (bottom panel) alone or in combination with $1 \mu \mathrm{M}$ HpTx as indicated.

Perfusion of the cells with $50 \mu \mathrm{M}$ TEA in the bath solution led to a $24.02 \pm 4.6 \%$ reduction of the peak current amplitude, and $1 \mu \mathrm{M} \mathrm{HpTx}$ increased inhibition up to a $46.2 \pm 5.4 \%$. However, application of $500 \mu \mathrm{M}$ TEA completely eliminated the HpTx-sensitive current, suggesting that both drugs are acting on the same channel population. These data prompted us to explore the possibility that some accessory subunit of Kv4 channels with an extracellular domain could modify TEA binding to Kv4 channels in rabbit chemoreceptor cells.

\subsection{Presence of DPPX in Rabbit CB Chemoreceptor Cells}

We have used immunocytochemistry in isolated chemoreceptor cells to determine the presence at the protein level of DPPX, a Kv4 accessory subunit with a large C-terminal extracellular domain (Wada et al., 1992). We found that all TH-positive cells were stained with anti-DPPX antibody and also observed a perfect correlation with double-immunocytochemistry with 
antiKv4.3 and anti-DPPX antibodies (Figure 2A-B). To further confirm the presence of DPPX in rabbit $\mathrm{CB}$ chemoreceptor cells, qPCR experiments using SYBRgreen were performed in pooled chemoreceptor cells obtained from CB cultures, using RPL18 as housekeeping gene (Kaab et al., 2005). We have also measured DPPX mRNA levels in granule cells from cerebellum. We found high expression levels of DPPX mRNA in our preparation (rabbit CB chemoreceptor cells) where the quantification shows a 50 -fold increase as compared to the control tissue (hippocampus), and almost ten times more than in granule cells (Figure 2C). Altogether, these data suggested that DPPX could represent a good candidate to modulate $\mathrm{Kv} 4$ currents in $\mathrm{CB}$ chemoreceptor cells.

A

B
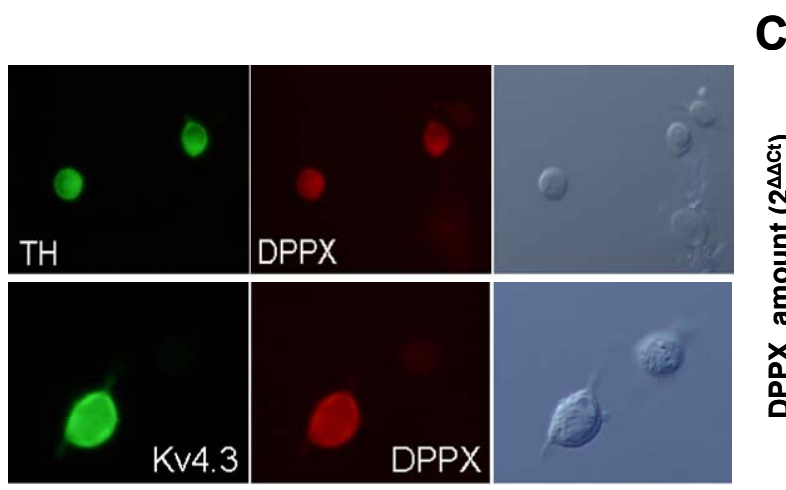

Figure 2. DPPX expression in rabbit chemoreceptor cells. A) Immunofluorescence labeling of DPPX shows the expression of DPPX in every TH-positive cell. B) Double labeling of $\mathrm{CB}$ chemoreceptor cells with anti-Kv4.3 and anti-DPPX antibodies shows an almost perfect coexpression of these two proteins. C) Real-time PCR showing the relative abundance of DPPX mRNA in CB chemoreceptor cells in primary culture. DPPX mRNA abundance in cerebellar granule cells (G cells) were determined for comparison. Each bar is the mean \pm SEM of four to seven individual determinations.

\subsection{DPPX Contribution to Transient Outward $\mathbf{K}^{+}$ Currents in Chemoreceptor Cells}

In order to determine whether TEA sensitivity of transient outward currents in rabbit $\mathrm{CB}$ chemoreceptor cells was in fact due to the presence of Kv4/DPPX heteromultimers, we studied the effects of functional suppression of DPPX on the kinetics and pharmacology of the native currents by using siRNA against rabbit DPPX.

We introduced siRNA against rabbit DPPX in the chemoreceptor cells by using single-cell electroporation and analyzing the magnitude of the currents and their kinetics. We observed a slower time course of inactivation in the siRNA DPPX transfected cells, and the fit of the data to a biexponential decay function indicated that siRNA DPPX induced a decrease in the amplitude of the fast component of the current. Also, we found a significant 
DPPX Modifies TEA Sensitivity of the Kv4 Channels in Rabbit Carotid Body Chemoreceptor Cells.

reduction in the proportion of the transient component of the current, which is in agreement with the well-known chaperone function of DPP proteins on Kv4 currents (Jerng et al., 2005;Nadal et al., 2003).
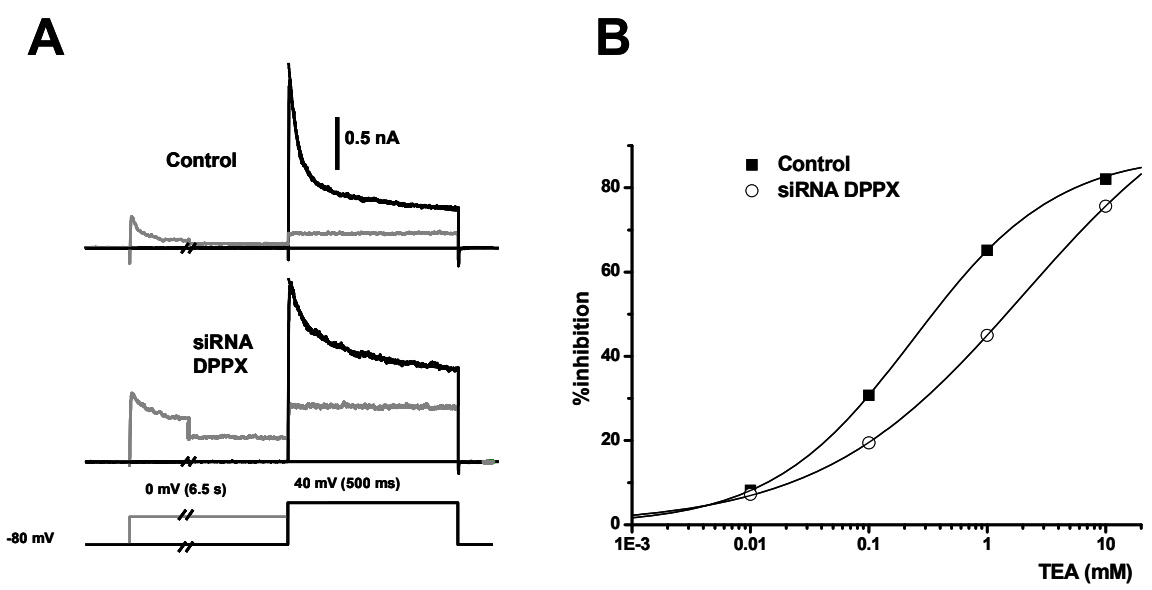

Figure 3. Effect of DPPX down-regulation in the transient $\mathrm{K}^{+}$current in rabbit chemoreceptor cells. A) Currents recorded from two individual CB chemoreceptor cells in control and after electroporation of siRNA DPPX, with a voltage protocol in which 500 $\mathrm{ms}$ depolarizing steps to $+40 \mathrm{mV}$ follow $6.5 \mathrm{~s}$ prepulses to two different potentials, -80 $\mathrm{mV}$ (black trace) and $0 \mathrm{mV}$ (grey trace). The difference between the current amplitude at $+40 \mathrm{mV}$ in these two pulses is defined as the transient outward current. The black thick line shows the fit of the currents to a double-exponential function. B) The effect of TEA in two individual CB chemoreceptor cells in control and after electroporation of siRNA DPPX is represented as percentage of inhibition, and was calculated from the reduction in the peak current amplitude in depolarizing pulses to $+40 \mathrm{mV}$. The continuous lines show the fit of the data to a hyperbolic function with one binding site for TEA. The half inhibitory concentration in control cell is $240 \mu \mathrm{M}$ and in siRNA DPPX transfected cell 2 $\mathrm{mM}$.

Once we obtained functional evidence of DPPX down-regulation in chemoreceptor cells, we explored if there was some effect on the TEA sensitivity of the transient current. In each cell studied, we applied 4 different TEA concentrations (from $10 \mu \mathrm{M}$ to $10 \mathrm{mM}$ ) and we normalized the reduction in the peak current amplitude observed in depolarizing pulses to $+40 \mathrm{mV}$. The dose-response curve obtained in two individual CB chemoreceptor cells in control and after electroporation of siRNA DPPX is depicted in Figure 3B. We found a significant reduction of TEA sensitivity in siRNA DPPX transfected cells in the intermediate range of TEA concentrations ( 0.1 and $1 \mathrm{mM})$ with no changes at lower and higher concentrations (Figure 3B). The average fit of the data to a hyperbolic function shows that down-regulation of DPPX leads to a 3-fold decrease in the TEA affinity of the current (half inhibitory concentration of $189.76 \pm 13.44 \mu \mathrm{M}$ in control cells versus $540.96 \pm 40.84 \mu \mathrm{M}$ in siRNA DPPX transfected cells). 


\section{DISCUSION}

The data presented in this work allow us to propose that the sensitivity to TEA of the Kv4 channels that largely contribute to the transient outward $\mathrm{K}^{+}$ currents of rabbit CB chemoreceptor cells is related to the presence of DPPX accessory subunits in these cells. These findings provide an explanation to our previous observation regarding TEA sensitivity of rabbit $\mathrm{CB} \mathrm{K}_{\mathrm{O} 2}$ and are also relevant to understand the contribution of DPPX to the functional properties of transient $\mathrm{K}^{+}$currents in this preparation.

The tissue-specific assembly of the Kv4 multiproteic complex could explain TEA-sensitivity of Kv4 channels in rabbit CB chemoreceptors. It has been proposed that both the relative abundance of DPPX as well as the cellspecific expression of the DPPX splice isoforms could confer Kv4 channels with differential modulation at various levels. In this regard, it is noteworthy that the expression levels of DPPX are ten times higher in CB chemoreceptor cells than in cerebellar granule cells, a preparation in which DPPX has been found to be prominently expressed (Nadal et al., 2003;Nadal et al., 2006).

The data obtained allow us to conclude that many of the modulatory effects of DPPX on Kv4 currents described in heterologous expression system are also relevant for native currents. However, it must be taken into account that these effects are muffled because the transient $\mathrm{K}^{+}$current component does not reflect an homogeneous channel population (Sanchez et al., 2002;LópezLópez et al., 2003;Kaab et al., 2005), and because siRNA against DPPX does not produce a complete knock out of DPPX subunit expression.

In spite of these limitations, we can extract relevant and unambiguous conclusions from the data obtained from siRNA DPPX transfected cells regarding the role of DPPX subunit in rabbit CB chemoreceptor cells: 1) DPPX has a chaperone role on Kv4 currents, as in the presence of siRNA DPPX there is a significant decrease in the current density of the transient component, 2) DPPX accelerates the time course of inactivation of the channels, as DPPX down-regulation decreases the proportion of the fast component of the current, and 3) DPPX contributes to TEA sensitivity of transient $\mathrm{K}^{+}$current, as siRNA DPPX transfected cells exhibited a significant decrease of their TEA sensitivity. With regards to this latter observation, it is interesting to point out that the change in TEA sensitivity was not observed along the whole range of TEA concentrations, but only for the high micromolar-low millimolar ones, which in all likelihood represent the Kv4 component of the outward $\mathrm{K}^{+}$current (component B2 in Figure 1). We have previously described that the component blocked by lower TEA concentrations $(1-50 \mu \mathrm{M})$ represents Kv3.4 channels (Sanchez et al., $2002 ; \mathrm{Kaab}$ et al., 2005), and this component is not modified by siRNA DPPX, but is reduced by down-regulation of Kv3.4 channels (as under chronic hypoxia stimulation (Kaab et al., 2005), and selectively abolished with transfection of siRNA against rabbit Kv3.4 (unpublished observation). On the other hand, at high TEA concentrations the fraction of the current blocked is a non-inactivating one, that we have evidences indicating that is 
DPPX Modifies TEA Sensitivity of the Kv4 Channels in Rabbit Carotid Body Chemoreceptor Cells.

carried by members of the Kv1 family of channels (Perez-Garcia et al., 2000).

Altogether, this set of data indicates that the effect of siRNA DPPX is very selective on Kv4 currents and that DPPX association contributes to the atypical pharmacological profile of $\mathrm{Kv} 4$ currents in rabbit $\mathrm{CB}$ chemoreceptor cells.

\section{ACKNOWLEDGMENTS}

We thank Esperanza Alonso for excellent technical assistance. This work was supported by Ministerio de Sanidad y Consumo, Instituto de Salud Carlos III grants R006/009 (Red Heracles) and PI041044 (JRLL), Ministerio de Educación y Ciencia grant BFU2004-05551 (MTPG) and Junta de Castilla y León grant VA011C05.

\section{REFERENCES}

Colinas, O., Perez-Carretero, F. D., Lopez-Lopez, J. R., \& Perez-Garcia, M. T. (2008). A Role for DPPX Modulating External TEA Sensitivity of Kv4 Channels. The Journal of General Physiology 131, 455-471.

Jerng, H. H. \& Covarrubias, M. (1997). K+ channel inactivation mediated by the concerted action of the cytoplasmic N- and C-terminal domains. Biophysical Journal 72, 163174.

Jerng, H. H., Kunjilwar, K., \& Pfaffinger, P. J. (2005). Multiprotein assembly of Kv4.2, KChIP3 and DPP10 produces ternary channel complexes with ISA-like properties. $J$ Physiol 568, 767-788.

Kaab, S., Miguel-Velado, E., López-López, J. R., \& Perez-Garcia, M. T. (2005). Down regulation of kv3.4 channels by chronic hypoxia increases acute oxygen sensitivity in rabbit carotid body. $J$ Physiol.

Liu, L. Y., Hoffman, G. E., Fei, X. W., Li, Z., Zhang, Z. H., \& Mei, Y. A. (2007). Delayed rectifier outward $\mathrm{K}+$ current mediates the migration of rat cerebellar granule cells stimulated by melatonin. J Neurochem. 102, 333-344.

Livak, K. J. \& Schmittgen, T. D. (2001). Analysis of relative gene expression data using realtime quantitative PCR and the 2(-Delta Delta C(T)) Method. Methods 25, 402-408.

López-López, J. R., De Luis, D. A., \& Gonzalez, C. (1993). Properties of a transient K current in chemoreceptor cells of rabbit carotid body. J.Physiol.Lond. 460, 15-32.

López-López, J. R., Gonzalez, C., \& Pérez-García, M. T. (1997). Properties of ionic currents from isolated adult rat carotid body chemoreceptor cells: effect of hypoxia. J.Physiol 499, 429-441.

López-López, J. R., Perez-Garcia, M. T., Sanz-Alfayate, G., Obeso, A., \& Gonzalez, C. (2003). Functional identification of Kvalpha subunits contributing to the O2sensitive $\mathrm{K}+$ current in rabbit carotid body chemoreceptor cells. Adv.Exp.Med.Biol. 536:33-9., 33-39.

Martina, M., Schultz, J. H., Ehmke, H., Monyer, H., \& Jonas, P. (1998). Functional and molecular differences between voltage-gated $\mathrm{K}+$ channels of fast-spiking interneurons and pyramidal neurons of rat hippocampus. J.Neurosci. 18, 8111-8125.

Nadal, M. S., Ozaita, A., Amarillo, Y., Vega-Saenz, d. M., Ma, Y., Mo, W., Goldberg, E. M., Misumi, Y., Ikehara, Y., Neubert, T. A., \& Rudy, B. (2003). The CD26-related dipeptidyl aminopeptidase-like protein DPPX is a critical component of neuronal Atype K+ channels. Neuron 37, 449-461. 


\section{(O. Colinas et al.)}

Nadal, M. S., Amarillo, Y., de Miera, E. V.-S., \& Rudy, B. (2006). Differential characterization of three alternative spliced isoforms of DPPX. Brain Research 1094, 1-12.

Pak, M. D., Baker, K., Covarrubias, M., Butler, A., Ratcliffe, A., \& Salkoff, L. (1991). mShal, a subfamily of A-type $\mathrm{K}+$ channel cloned from mammalian brain. Proc.Natl.Acad.Sci.U.S.A 88, 4386-4390.

Perez-Garcia, M. T., Colinas, O., Miguel-Velado, E., Moreno-Dominguez, A., \& LópezLópez, J. R. (2004). Characterization of the Kv channels of mouse carotid body chemoreceptor cells and their role in oxygen sensing. Journal of Physiology-London $557,457-471$.

Perez-Garcia, M. T., López-López, J. R., Riesco, A. M., Hoppe, U. C., Marban, E., Gonzalez, C., \& Johns, D. C. (2000). Viral gene transfer of dominant-negative Kv4 construct suppresses an $\mathrm{O} 2$-sensitive $\mathrm{K}+$ current in chemoreceptor cells. J.Neurosci. 20, 56895695.

Pérez-García, M. T., Obeso, A., López-López, J. R., Herreros, B., \& Gonzalez, C. (1992). Characterization of cultured chemoreceptor cells dissociated from adult rabbit carotid body. AJP - Lung Cellular and Molecular Physiology 263, C1152-9.

Rae, J. L. \& Levis, R. A. (2002). Single-cell electroporation. Pflugers Arch. 443, 664-670.

Sanchez, D., López-López, J. R., Perez-Garcia, M. T., Sanz-Alfayate, G., Obeso, A., Ganfornina, M. D., \& Gonzalez, C. (2002). Molecular identification of Kvalpha subunits that contribute to the oxygen-sensitive $\mathrm{K}(+)$ current of chemoreceptor cells of the rabbit carotid body. J.Physiol 542, 369-382.

Sanguinetti, M. C., Johnson, J. H., Hammerland, L. G., Kelbaugh, P. R., Volkmann, R. A., Saccomano, N. A., \& Mueller, A. L. (1997). Heteropodatoxins: peptides isolated from spider venom that block Kv4.2 potassium channels. Mol.Pharmacol. 51, 491498.

Song, W. J., Tkatch, T., Baranauskas, G., Ichinohe, N., Kitai, S. T., \& Surmeier, D. J. (1998). Somatodendritic depolarization-activated potassium currents in rat neostriatal cholinergic interneurons are predominantly of the A type and attributable to coexpression of Kv4.2 and Kv4.1 subunits. J.Neurosci. 18, 3124-3137.

Wada, K., Yokotani, N., Hunter, C., Doi, K., Wenthold, R. J., \& Shimasaki, S. (1992). Differential expression of two distinct forms of mRNA encoding members of a dipeptidyl aminopeptidase family. Proc.Natl.Acad.Sci U S.A 89, 197-201.

Wada, K., Yokotani, N., Hunter, C., Doi, K., Wenthold, R. J., \& Shimasaki, S. (1992b). Differential expression of two distinct forms of mRNA encoding members of a dipeptidyl aminopeptidase family. Proc.Natl.Acad.Sci U S.A 89, 197-201. 